\title{
The relationship between functional levels and fear-avoidance beliefs following anterior cruciate ligament reconstruction
}

\author{
Michael D. Ross
}

Received: 27 August 2009/Accepted: 1 November 2010/Published online: 30 November 2010

(C) The Author(s) 2010. This article is published with open access at Springerlink.com

\begin{abstract}
Background The purpose of this study is to assess the relationship between functional levels in activities of daily living and sports and fear-avoidance beliefs in patients with a history of anterior cruciate ligament reconstruction (ACLR), after controlling for injury-related variables and physical impairment measures.

Patients and methods Forty-eight subjects (34 men, 14 women; age $20.6 \pm 1.2$ years), at a mean of $31.7 \pm 16.2$ months following ACLR, participated in this study. Functional levels in activities of daily living and sports were assessed with the Knee Outcome Survey (KOS) Activities of Daily Living Scale (ADLS) and Sports Activity Scale (SAS). Fear-avoidance beliefs were assessed with the physical activity subscale of the fear-avoidance beliefs questionnaire (FABQ), which was adapted for use in patients with knee pathology. Injury-related variables included whether or not additional knee surgery was performed after the initial ACLR and the number of months from the most recent ACLR to participation in this study. Physical impairment measures included single-leg hop capabilities, quadriceps strength, and anterior knee joint laxity.

Results Hierarchical linear regression analysis revealed that scores on the physical activity subscale of the FABQ
\end{abstract}

The opinions expressed herein are those of the authors and do not necessarily reflect the opinions of the Department of Defense, the United States Air Force, or other federal agencies.

\section{D. Ross}

U.S. Air Force Physical Medicine Training Programs,

Fort Sam Houston, TX, USA

M. D. Ross ( $\square)$

598 American Flag, Cibolo, TX 78101, USA

e-mail: romoross@msn.com contributed significantly to KOS ADLS and SAS scores after accounting for injury-related variables and physical impairment measures. The final regression model accounted for $61 \%$ of the variance in KOS ADLS and SAS scores $(P<0.001)$.

Conclusion These results suggest that fear-avoidance beliefs following ACLR can potentially adversely influence functional levels in activities of daily living and sports.

Keywords Knee surgery - Outcomes · Rehabilitation · Prognosis

\section{Introduction}

Following anterior cruciate ligament reconstruction (ACLR), it is commonly recommended to assess the status of different physical impairments [1-3], presumably because these factors provide some estimate of functional levels following ACLR. Examples of physical impairments include single-leg hop ability, quadriceps strength, and anterior tibiofemoral joint laxity. Psychological variables have also been shown to influence functional levels following ACLR [4-7]. An example of a psychological impairment that may influence disability is fear-avoidance beliefs [8], which relates to the fear of painful reinjury that patients with a history of ACLR may experience, and the role of fear in causing patients to avoid behaviors that may potentially elicit pain or reinjury.

Kvist et al. [5] investigated whether fear of reinjury is a significant factor for returning to preinjury levels of activity in patients following ACLR. While only 53\% of their patients returned to their preinjury level of activity at 4 years after ACLR, they determined that the patients who did not 
return to preinjury activity levels had greater fear of reinjury, and this high fear of reinjury was correlated with low kneerelated quality of life. Chmielewski et al. [4] assessed fear of movement/reinjury levels and determined the association with function at different timeframes during ACLR rehabilitation. They determined that, while fear of movement/ reinjury levels appear to decrease during ACLR rehabilitation, these factors were still associated with function in the timeframe when patients return to sports. While Kvist et al. [5] and Chmielewski et al. [4] both determined that fear of movement/reinjury are supported as determinants of function after ACLR, neither assessed the influence of relevant impairments routinely assessed in clinical practice (i.e., single-leg hop capabilities, quadriceps strength, and anterior knee joint laxity) in conjunction with fear of movement and reinjury in their patient populations.

Generally, ACLR is perceived to be a successful procedure; however, critical analysis has shown that clinical outcomes are less than optimal, as a recent meta-analysis has shown that normal knee function is restored in only $37 \%$ of patients undergoing ACLR [9]. Understanding which physical or psychological impairments contribute most significantly to disability following ACLR will assist in establishing appropriate rehabilitation programs in this patient population to ensure that functional levels are optimized following surgery. This may be especially important for fear-avoidance beliefs, which are not consistently addressed despite clinicians acknowledging fear of reinjury as the main injury-related psychological concern following injury [10].

The purpose of this study is to assess the relationship between functional levels in activities of daily living and sports and fear-avoidance beliefs in patients with a history of ACLR, after controlling for injury-related variables and physical impairment measures. It was hypothesized that fear-avoidance beliefs would contribute to functional levels in activities of daily living and sports, after controlling for injury-related variables and physical impairment measures.

\section{Patients and methods}

\section{Subjects}

Forty-eight subjects (34 men, 14 women) participated in this study (age $20.6 \pm 1.2$ years, height $177.3 \pm 9.1 \mathrm{~cm}$, weight $77.9 \pm 12.5 \mathrm{~kg}$ ). At the time of this study, all subjects were enrolled at the US Air Force Academy, CO. While subjects were not intercollegiate athletes, they participated in mandatory intramural athletics and military training 4-7 days/week prior to their knee injury.

Selection criteria for this study included history of unilateral ACLR performed greater than 12 months from time of participation in this study, completion of a rehabilitation program that focused on full return to military and athletic activities, and clearance from an orthopedic surgeon to return to preinjury activity levels following ACLR. Prior to participation, all subjects read and signed an informed consent document approved by the Institutional Review Board at the US Air Force Academy, CO. This study was in accordance with the ethical standards of the 1964 Declaration of Helsinki as revised in 2000.

All subjects had initially undergone ACLR using a bonepatellar tendon-bone autograft. Mean time from injury to initial ACLR for subjects in this study was $31.3 \pm 28.5$ days (range 10-154 days). Six subjects (13\%) underwent revision ACLR, in which their ipsilateral distal semitendinosus and gracilis tendons were doubled and used for reconstruction. None of the six subjects who underwent revision ACLR had undergone any other knee surgeries. However, six other subjects had additional knee surgery at a mean of $17.2 \pm 11.8$ months (range 3-37 months) following ACLR, including partial meniscectomy $(\mathrm{n}=5)$ and diagnostic arthroscopy $(\mathrm{n}=1)$. Subjects were tested at a mean of $28.3 \pm 15.1$ months (range 12-69 months) following their most recent knee surgery. At time of testing, all subjects had resumed all required military and athletic activities.

\section{Research design}

This was a descriptive study that assessed the relationship between functional levels in activities of daily living and sports and fear-avoidance beliefs in patients with history of ACLR, after controlling for injury-related variables and physical impairments. The dependent variable was functional levels in activities of daily living and sports as measured by the combined scores on the Knee Outcome Survey (KOS) Activities of Daily Living Scale (ADLS) and Sports Activity Scale (SAS) [11, 12]. The independent variables were fear-avoidance beliefs, injury-related variables, and physical impairments. Fear-avoidance beliefs were assessed with a version of the physical activity subscale of the fear-avoidance beliefs questionnaire (FABQ) that was modified for the knee; the FABQ was originally used to assess fear-avoidance beliefs in patients with lowback pain. Injury-related variables in this study included whether or not the patient underwent additional surgery following initial ACLR and time from ACLR to testing. Physical impairment measures included single-leg hop for distance test performance, isokinetic quadriceps function, and anterior tibiofemoral joint laxity.

Functional measure

The degree of functional limitations experienced by the subjects in this study was assessed through the combined 
scores on the KOS ADLS and SAS [11, 12]. Items on the ADLS [12] relate to symptoms and functional limitations that individuals may experience during activities of daily living. Items on the SAS [11] assess symptoms and functional limitations that individuals may experience while playing sports. Responses regarding symptoms were graduated in terms of the amount of participation restrictions that individuals experienced during activities of daily living or sports due to the symptom. Responses regarding functional limitations were graduated from no limitation to inability to perform the specific activity.

The KOS ADLS and SAS were numerically graded on a scale of $0-100$, with higher scores indicating higher functional levels [11, 12]. The combined scores of the KOS ADLS and SAS (possible range 0-200) were used for data analysis. The KOS has been shown to be a reliable and valid measure of disability in patients with knee impairments [11, 12].

Fear-avoidance beliefs questionnaire

Fear-avoidance beliefs were measured using the physical activity subscale of the FABQ. The physical activity subscale of the FABQ quantifies the level of fear about physical activity and has primarily been studied in patients with low-back pain. The FABQ also has a work subscale, which quantifies the level of fear about workrelated activities. The work subscale of the FABQ was not used as a method of assessment in this study, as the subjects were full-time students who were not employed. The instrument consists of five items, which are scored from 0 to 6 . Possible scores range from 0 to 30 , with higher scores representing increased fear-avoidance beliefs. Previous studies have reported good reliability for the FABQ in patients with low-back pain [13]. To apply the FABQ in patients following ACLR, we adapted the form to use in patients with knee pathology as described by Piva et al. [14] and van Baer et al. [15]. We changed the descriptors of physical activities such as bending, lifting, walking, or driving to physical activities such as walking, running, kneeling, or driving, and changed the word "back" to "knee" throughout the form. Prior to the descriptive study, we determined the test-retest reliability for the FABQ to be high (intraclass correlation coefficient $=0.92$ ).

Injury-related variables

\section{Knee surgeries after initial anterior cruciate ligament reconstruction}

For each subject, it was documented whether or not additional knee surgery was performed after the initial ACLR.
Time from anterior cruciate ligament reconstruction to testing

The number of months from the most recent ACLR to participation in this study was calculated for each subject.

Physical impairment measures

\section{Single-leg hop test for distance}

A tape measure was secured to the floor, and subjects stood on the leg being tested with the anterior aspect of their athletic shoe at the zero mark of the tape measure. While standing on one leg, subjects were instructed to hop as far as possible forward and land on the same leg. The distance hopped was then measured from the zero mark of the tape measure to the point where the subject's heel hit the ground. No other biomechanical data were recorded. Following one practice trial, subjects performed three test trials for each lower extremity in alternating fashion, beginning with the noninvolved lower extremity. Based upon the average of the three test trials, single-leg hop test indices were calculated and used for data analysis. These indices were calculated by expressing the scores of the involved lower extremity as a percentage of the noninvolved lower extremity (i.e., involved lower extremity score/noninvolved lower extremity score $\times 100$ ).

\section{Isokinetic quadriceps test}

Quadriceps peak torque was assessed with the kinetic communication isokinetic dynamometer (Chattanooga Group Inc., Hixson, TN). With subjects in a seated position, concentric isokinetic knee extension was tested at $60 \%$ $\mathrm{s}$ between $90^{\circ}$ and $0^{\circ}$ of flexion. Subjects were requested to complete five maximal test repetitions for each lower extremity. Quadriceps peak torque indices were calculated and used for data analysis. These indices were calculated by expressing the involved lower extremity scores as a percentage of the noninvolved lower extremity (i.e., involved lower extremity score/noninvolved lower extremity score $\times 100$ ).

\section{Anterior tibiofemoral joint laxity test}

Anterior tibiofemoral joint laxity was assessed with the KT-1000 knee joint arthrometer (MEDmetric Corp., San Diego, CA) while subjects were supine with the knee positioned in $20^{\circ}$ to $30^{\circ}$ of flexion. With the KT-1000 strapped to the leg, one hand of the examiner stabilized the thigh and patellar pad of the KT-1000 and the other hand of 
the examiner applied a maximal manual anteriorly directed force on the proximal calf. The amount of movement was quantified in millimeters. Three test trials were performed on each knee of the subject, with the maximum value of the three test trials used to calculate side-to-side differences (the score of the involved knee-the score of the noninvolved knee), which were used for data analysis.

\section{Procedure}

Prior to the descriptive study, test-retest reliability for the measurement procedures used in this study were calculated and determined to be acceptable in subjects with a history of ACLR [16]. Following a medical chart review, which determined the date and type of previous knee surgeries, subjects were assessed during a single testing session in the following manner: completion of the FABQ and the KOS ADLS and SAS followed by KT-1000 testing.

Each subject then completed a warm-up, which consisted of 5 min of self-paced stationary cycling, followed by quadriceps, hamstring, and calf muscle stretching of both lower extremities. Following the warm-up, isokinetic testing of the quadriceps took place followed by single-leg hop testing. For KT-1000, isokinetic, and single-leg hop testing, the noninvolved lower extremity was tested prior to the involved lower extremity. A single investigator collected all data.

\section{Data analysis}

A hierarchical linear regression analysis assessed the relationship between patient-reported functional levels in activities of daily living and sports and fear-avoidance beliefs after controlling for injury-related variables and physical impairment measures. Scores on the Knee Outcome Survey served as the dependent variable. Scores on the physical activity subscale of the FABQ, injury-related variables, and physical impairment measures served as the independent variables. Data pertaining to injury-related variables were entered in the first step of the regression analysis, physical impairment measures were entered in the second step of the regression analysis, and scores on the physical activity subscale of the FABQ were entered in the third step of the regression analysis. Developing a regression model in this way provides a means to analyze the unique contribution of the physical activity subscale of the FABQ, after controlling for the contribution of injuryrelated variables and physical impairment measures. This method of analysis is consistent with previous studies evaluating the influence of fear avoidance on function and disability [4, 17]. Statistical significance was set at $P<0.05$.

\section{Results}

Means, standard deviations, and ranges for the dependent and independent variable(s) used in the descriptive study are presented Table 1. The results of the hierarchical linear regression analysis are presented in Table 2. Injury-related variables (whether or not the subject had additional knee surgery after the initial ACLR and the number of months from initial ACLR to participation in this study) collectively contributed $48 \%$ of the variance in functional scores as measured by the combined scores of the KOS ADLS and SAS $(P<0.001)$. Physical impairment measures (singleleg hop for distance test performance, isokinetic quadriceps function, and anterior tibiofemoral joint laxity) contributed an additional $1 \%$ of the variance $(P<0.001)$. Finally, scores on the physical activity subscale of the FABQ contributed an additional $12 \%$ of the variance $(P<0.001)$, after accounting for injury-related variables and physical impairment measures.

The final regression model accounted for $61 \%$ of the variance in functional scores as measured by the combined scores of the KOS ADLS and SAS $(P<0.001)$. The only significant factors in the final regression model were whether or not the subject had additional knee surgery after the initial ACLR and FABQ physical activity subscale scores, and the regression coefficients indicated that not having additional knee surgery after the initial ACLR and lower fear-avoidance beliefs (i.e., lower scores on the physical activity subscale of the FABQ) were associated

Table 1 Means, standard deviations, and ranges for the dependent and independent variable(s)

\begin{tabular}{lcl}
\hline & Mean \pm SD & Range \\
\hline Dependent variable & & \\
Knee Outcome Survey & $176.98 \pm 23.74$ & $86.75-200$ \\
Activities of Daily Living Scale & $90.73 \pm 9.38$ & $58.75-100$ \\
Sports Activity Scale & $86.25 \pm 14.83$ & $28-100$ \\
Independent variables & & \\
$\quad$ Time from ACLR to testing & $31.73 \pm 16.24$ & $12-72$ \\
(months) & & \\
Single-leg hop index & $98.06 \pm 5.42$ & $78.26-109.72$ \\
Quadriceps peak torque index & $93.38 \pm 13.07$ & $70.77-126.25$ \\
KT-1000 (mm) & $2.00 \pm 2.83$ & $-4.00-8.00$ \\
FABQ & $12.88 \pm 5.26$ & $6-25$ \\
\hline
\end{tabular}

Knee Outcome Survey, combined scores for Knee Outcome Survey Activities of Daily Living Scale and Sports Activity Scale; ACLR anterior cruciate ligament reconstruction; single-leg hop index and quadriceps peak torque index, involved lower extremity scores/noninvolved lower extremity scores $\times 100$; KT-1000, involved lower extremity scores-noninvolved lower extremity scores; $F A B Q$ physical activity subscale scores of the fear-avoidance beliefs questionnaire; $S D$ standard deviation 
Table 2 Results of the hierarchical linear regression analysis - the dependent variable was functional levels in activities of daily living and sports as measured by Knee Outcome Survey scores
$A C L R$ anterior cruciate ligament reconstruction

* $P$ value for model at each step, ** $P$ value for each variable at each step

\begin{tabular}{lccc}
\hline Independent variable(s) & Parameter estimate & Standard error & $P$ value** \\
\hline Step 1 & & & \\
Knee surgery after initial ACLR & -33.06 & 5.43 & $<0.001$ \\
Time from ACLR to testing & 0.47 & 0.15 & $<0.001$ \\
Adjusted $R^{2}=0.48, P<0.001^{*}$ & & & \\
Step 2 & & 5.46 & $<0.001$ \\
Knee surgery after initial ACLR & -30.99 & 0.16 & 0.011 \\
Time from ACLR to testing (months) & 0.43 & 0.47 & 0.048 \\
Single-leg hop index & 0.99 & 0.21 & 0.87 \\
Quadriceps peak torque index & 0.03 & 0.89 & 0.70 \\
KT-1000 & -0.35 & & \\
Adjusted $R^{2}=0.49, P<0.001^{*}$ & & & \\
Step 3 & & 5.11 & 0.26 \\
Knee surgery after initial ACLR & -24.78 & 0.16 & 0.73 \\
Time from ACLR to testing (months) & 0.18 & 0.47 & 0.96 \\
Single-leg hop index & 0.34 & 0.19 & 0.86 \\
Quadriceps peak torque index & -0.01 & 0.79 & $<0.001$ \\
KT-1000 & -0.14 & 0.54 & \\
FABQ & -1.96 & & \\
Adjusted $R^{2}=0.61, P<0.001^{*}$ & & & \\
\hline
\end{tabular}

with higher functional levels in activities of daily living and sports (i.e., higher scores on the KOS ADLS and SAS).

\section{Discussion}

The purpose of this study is to assess the relationship between functional levels in activities of daily living and sports and fear-avoidance beliefs in patients with a history of ACLR, after controlling for injury-related variables and physical impairment measures. It was hypothesized that fear-avoidance beliefs would contribute to functional levels in activities of daily living and sports, after controlling for injury-related variables and physical impairment measures. This hypothesis was based, in part, on the results of Kvist et al. [5] and Chmielewski et al. [4], who determined that fear of movement/reinjury are determinants of function after ACLR. This hypothesis was also based upon the implications of ACLR in the population assessed in this study. At the time of this study, each subject was a student in a military academy. At this institution, if a student does not successfully rehabilitate following ACLR, graduation and the ability to secure certain military positions following graduation could be jeopardized. The results of this study suggest that fear-avoidance beliefs following ACLR can potentially adversely influence functional levels in activities of daily living and sports.

While Kvist et al. [5] and Chmielewski et al. [4] both previously determined that fear of movement/reinjury are supported as determinants of function after ACLR, neither assessed the influence of relevant impairments routinely assessed in clinical practice in conjunction with fear of movement and reinjury in their patient populations. The results of this study suggest that fear-avoidance beliefs explained a significant amount of the variance in functional performance levels in activities of daily living and sports even after controlling for injury-related variables and physical impairment measures. Specifically, scores on the physical activity subscale of the FABQ contributed an additional $12 \%$ of the variance in functional performance levels in activities of daily living and sports, after accounting for injury-related variables and physical impairment measures. The final regression model accounted for $61 \%$ of the variance in functional scores as measured by the combined scores of the KOS ADLS and SAS $(P<0.001)$.

Injury-related variables (whether or not the subject had additional knee surgery after the initial ACLR and the number of months from the most recent ACLR to participation in this study) were entered in the first step of the regression analysis and collectively contributed approximately $48 \%$ of the variance in functional scores as measured by the combined scores of the KOS ADLS and SAS. While the design of this study did not allow us to determine if fear-avoidance beliefs decrease over time following ACLR and if diminished fear-avoidance beliefs were associated with improved functional performance in activities of daily living and sports, other researchers have determined that fear of movement/reinjury levels were lower with increased time from ACLR [4]. Physical 
impairment measures (single-leg hop for distance test performance, isokinetic quadriceps function, and anterior tibiofemoral joint laxity as measured with the KT-1000) were entered in the second step of the regression analysis and contributed only an additional $1 \%$ of the variance, suggesting that the physical impairment measures did not substantially add to our ability to estimate patient-reported functional performance levels. Although the subjects in this study exhibited deficits for the involved lower extremity for the single-leg hop index (1.9\% deficit) and quadriceps peak torque test $(6.4 \%$ deficit), these deficits were small and may not have been great enough to influence functional performance levels in activities of daily living and sports. Perhaps there is a threshold for the single-leg hop index and quadriceps peak torque test scores, above which patients function normally and below which patients experience functional performance limitations of varying degrees [16]. While the KT-1000 may provide valuable information for diagnosis of a ruptured anterior cruciate ligament, it does not adequately assess the functional capabilities of the knee [18] and it serves as a limited measure of knee function during dynamic weight-bearing activities routinely performed during activities of daily living and sports following ACLR.

While our findings support the preliminary notion that fear-avoidance beliefs could adversely influence functional levels in patients following ACLR, it is well established that fear-avoidance beliefs play a significant role in the development of chronic disability in patients with low-back pain. Fear-avoidance behaviors can exacerbate pain intensity and pain-related disability $[19,20]$ and lead to deconditioning and less effective coping strategies [21]. For patients with elevated fear-avoidance beliefs, reducing fear of reinjury becomes a rehabilitation goal that is achieved through patient education and graded exercise prescription [22]. The results of this study, as well as others, suggest that research efforts should be made in determining the effect of interventions geared toward addressing fearavoidance beliefs in patients following ACLR on factors such as reinjury rates and disability levels.

There are limitations to this study that are worth mentioning. First, the study population was not homogeneous. We pooled subjects with history of single ACLR with subjects with history of revision ACLR into the analysis, and these groups may differ in their ability to successfully return to high activity levels following surgery, with revision ACLR being associated with lower activity levels [23], presumably due to both physical and psychological factors. Second, this study design did not include a followup evaluation after subjects were initially assessed. Followup evaluations would be needed to determine if the patients in this study experienced diminished fear-avoidance beliefs over time and if diminished fear-avoidance beliefs were associated with improved functional performance in activities of daily living and sports and decreased reinjury rates. Third, the FABQ was our only measure of psychological impairment. Further research should assess the influence of other psychological variables (i.e., depression, anxiety, heightened concern about illness, anger) on functional levels in patients following ACLR. Fourth, caution should be used in generalizing these results to other patient populations who may return to different activity levels following different types of knee surgery. All subjects in this study were enrolled in a military academy and had initially undergone ACLR in which a bone-patellar tendon-bone autograft was used. Additionally, subjects participated in similar supervised rehabilitation programs and returned to similar activity levels following surgery, which involved mandatory participation in intramural athletics and military training.

The results of this study indicated that fear-avoidance beliefs explained a significant amount of the variance in functional performance levels in activities of daily living and sports in patients following ACLR, even after controlling for injury-related variables and physical impairment measures. It is recommended that future research efforts determine the influence of demographic data (i.e., age, gender) and interventions geared toward addressing fear-avoidance beliefs in patients following ACLR. An emphasis should also be placed on ensuring that these interventions are implemented, as fear-avoidance beliefs are not consistently addressed despite clinicians acknowledging fear of reinjury as the main injury-related psychological concern following injury [10].

Acknowledgments The author would like to thank James J. Irrgang, PT, PhD, Craig R. Denegar, PT, PhD, and Constance M. McCloy, PT, EdD for their assistance with this project and the subjects who graciously participated in this study.

\section{Conflict of interest None.}

Open Access This article is distributed under the terms of the Creative Commons Attribution Noncommercial License which permits any noncommercial use, distribution, and reproduction in any medium, provided the original author(s) and source are credited.

\section{References}

1. Cascio BM, Culp L, Cosgarea AJ (2004) Return to play after anterior cruciate ligament reconstruction. Clin Sports Med 23:395-408

2. Myer GD, Paterno MV, Ford KR, Hewett TE (2008) Neuromuscular training techniques to target deficits before return to sport after anterior cruciate ligament reconstruction. J Strength Cond Res 22:987-1014

3. Myer GD, Paterno MV, Ford KR, Quatman CE, Hewett TE (2006) Rehabilitation after anterior cruciate ligament 
reconstruction: criteria-based progression through the return-tosport phase. J Orthop Sports Phys Ther 36:385-402

4. Chmielewski TL, Jones D, Day T, Tillman SM, Lentz TA, George SZ (2008) The association of pain and fear of movement/ reinjury with function during anterior cruciate ligament reconstruction rehabilitation. J Orthop Sports Phys Ther 38:746-753

5. Kvist J, Ek A, Sporrstedt K, Good L (2005) Fear of re-injury: a hindrance for returning to sports after anterior cruciate ligament reconstruction. Knee Surg Sports Traumatol Arthrosc 13:393-397

6. Lee DY, Karim SA, Chang HC (2008) Return to sports after anterior cruciate ligament reconstruction-a review of patients with minimum 5-year follow-up. Ann Acad Med Singapore 37:273-278

7. Vranceanu AM, Barsky A, Ring D (2009) Psychosocial aspects of disabling musculoskeletal pain. J Bone Joint Surg Am 91:2014-2018

8. Waddell G, Newton M, Henderson I, Somerville D, Main CJ (1993) A fear-avoidance beliefs questionnaire (FABQ) and the role of fear-avoidance beliefs in chronic low back pain and disability. Pain 52:157-168

9. Biau DJ, Tournoux C, Katsahian S, Schranz P, Nizard R (2007) ACL reconstruction: a meta-analysis of functional scores. Clin Orthop Relat Res 458:180-187

10. Mann BJ, Grana WA, Indelicato PA, O’Neill DF, George SZ (2007) A survey of sports medicine physicians regarding psychological issues in patient-athletes. Am J Sports Med 35:2140-2147

11. Borsa PA, Lephart SM, Irrgang JJ (1998) Sport-specificity of knee scoring systems to assess disability in anterior cruciate ligament-deficient athletes. J Sport Rehabil 7:44-60

12. Irrgang JJ, Snyder-Mackler L, Wainner RS, Fu FH, Harner CD (1998) Development of a patient reported measure of knee function. J Bone Joint Surg 80-A:1132-1145

13. Jacob T, Baras M, Zeev A, Epstein L (2001) Low back pain: reliability of a set of pain measurement tools. Arch Phys Med Rehabil 82:735-742
14. Piva SR, Fitzgerald GK, Irrgang JJ, Fritz JM, Wisniewski S, McGinty GT, Childs JD, Domenech MA, Jones S, Delitto A (2009) Associates of physical function and pain in patients with patellofemoral pain syndrome. Arch Phys Med Rehabil 90:285-295

15. van Baar ME, Dekker J, Oostendorp RA, Bijl D, Voorn TB, Lemmens JA, Bijlsma JW (1998) The effectiveness of exercise therapy in patients with osteoarthritis of the hip or knee: a randomized clinical trial. J Rheumatol 25:2432-2439

16. Ross MD, Irrgang JJ, Denegar CR, McCloy CM, Unangst ET (2002) The relationship between participation restrictions and selected clinical measures following anterior cruciate ligament reconstruction. Knee Surg Sports Traumatol Arthrosc 10:10-19

17. George SZ, Fritz JM, McNeil DW (2006) Fear-avoidance beliefs as measured by the fear-avoidance beliefs questionnaire: change in fear-avoidance beliefs questionnaire is predictive of change in self-report of disability and pain intensity for patients with acute low back pain. Clin J Pain 22:197-203

18. Harter RA, Osternig LR, Singer KM, James SL, Larson RL, Jones DC (1988) Long-term evaluation of knee stability and function following surgical reconstruction for anterior cruciate ligament insufficiency. Am J Sports Med 16:434-443

19. Vlaeyen JW, Kole-Snijders AM, Boeren RG, van Eek H (1995) Fear of movement/(re)injury in chronic low back pain and its relation to behavioral performance. Pain 62:363-372

20. Vlaeyen JW, Seelen HA, Peters M, de Jong P, Aretz E, Beisiegel E, Weber WE (1999) Fear of movement/(re)injury and muscular reactivity in chronic low back pain patients: an experimental investigation. Pain 82:297-304

21. McCracken LM (eds) (2005) Contextual cognitive behavioral therapy for chronic pain. IASP Press: Seattle, WA

22. Leeuw M, Goossens ME, Linton SJ, Crombez G, Boersma K, Vlaeyen JW (2007) The fear-avoidance model of musculoskeletal pain: current state of scientific evidence. J Behav Med 30:77-94

23. Dunn WR, Spindler KP, MOON Consortium (2010) Predictors of activity level 2 years after anterior cruciate ligament reconstruction (ACLR): a Multicenter Orthopaedic Outcomes Network (MOON) ACLR cohort study. Am J Sports Med 38:2040-2050 